\title{
Encapsulation of paclitaxel into a bio-nanocomposite. A study combining inelastic neutron scattering to thermal analysis and infrared spectroscopy
}

\author{
Murillo L. Martins ${ }^{1,2, a}$, Andrea Orecchini ${ }^{3,4}$, Luis Aguilera ${ }^{5}$, Juergen Eckert ${ }^{6}$, Jan Embs ${ }^{7}$, Aleksander Matic ${ }^{5}$, Margarida \\ J. Saeki ${ }^{2}$ and Heloisa N. Bordallo ${ }^{1, b}$ \\ ${ }^{1}$ Niels Bohr Institute, University of Copenhagen, 2100 Copenhagen, Denmark \\ ${ }^{2}$ Instituto de Biociências - Universidade Estadual Paulista - CP. 510, 18618-970 Botucatu - SP, Brazil \\ ${ }^{3}$ Institut Laue-Langevin, BP. 156, 38042 Grenoble, France \\ ${ }^{4}$ Dipartimento di Fisica, Università di Perugia, 06123 Perugia, Italy \\ ${ }^{5}$ Department of Applied Physics, Chalmers University of Technology, 41296 Göteborg, Sweden \\ ${ }^{6}$ Department of Chemistry, University of South Florida, 4202 E. Fowler Ave., Tampa, Florida 33620, USA \\ ${ }^{7}$ Laboratory for Neutron Scattering, Paul Scherrer Institute, 5232 Villigen, Switzerland
}

\begin{abstract}
The anticancer drug paclitaxel was encapsulated into a bio-nanocomposite formed by magnetic nanoparticles, chitosan and apatite. The aim of this drug carrier is to provide a new perspective against breast cancer. The dynamics of the pure and encapsulated drug were investigated in order to verify possible molecular changes caused by the encapsulation, as well as to follow which interactions may occur between paclitaxel and the composite. Fourier transformed infrared spectroscopy, thermal analysis, inelastic and quasi-elastic neutron scattering experiments were performed. These very preliminary results suggest the successful encapsulation of the drug.
\end{abstract}

\section{Introduction}

Paclitaxel $\left(\mathrm{C}_{47} \mathrm{H}_{51} \mathrm{NO}_{14}\right.$, hereafter PTX) is an anticancer drug whose effectiveness has been shown against different kinds of tumors [1,2], including breast cancer [3], Fig. 1. However, low water solubility and the fact that the drug damages healthy cells still limit its clinical application [4]. These issues can be minimized by encapsulating PTX into a bio-nanocomposite formed by a chitosan capsule modified by apatite crystals. The latter shows great affinity for breast cancer cells [5]. Additionally, by placing magnetic nanoparticles in the core of this bionanocomposite (hereafter bio-NCP) can allow for several further applications [6-9].

The synthesis of the bio-NCP itself has been recently reported [11]. Nevertheless, concerning the encapsulation, a number of questions remain open. For instance, it is well known that the PTX's anti-cancer activity is driven by its flexible structure [12-14], but the encapsulation can induce severe dynamical changes and interactions between the drug and the bio-NCP that may affect the releasing process. This work is the very first attempt to shed light into this particular problem.

To better understand the dynamics of pure and encapsulated PTX, Fourier transformed infrared spectroscopy (FTIR), thermal analysis, as well as inelastic (INS) and quasi-elastic neutron scattering (QENS) measurements were carried out [15]. Our results suggest that the drug

a e-mail: murillolongo@gmail.com

b e-mail: bordallo@nbi.ku can be successfully encapsulated. It seems however that the vibrations from the phenyl and acetyl groups are constrained. The in-vivo implications of this observation to the efficacy of this proposed drug carrier are out of the scope of this paper.

\section{Experimental section}

\subsection{Samples preparation}

All chemicals used for the preparation of the samples were purchased from Sigma-Aldrich.

The bio-NCP was prepared following the steps described in [11], with magnetic nanoparticles presenting the nominal formula $\mathrm{Mn}_{0.75} \mathrm{Zn}_{0.25} \mathrm{Fe}_{1.70} \mathrm{O}_{4}$, synthesized in a $0.1 \mathrm{M} \mathrm{NaOH}$ boiling solution, after a 120 min reaction. In order to modify the chitosan surface with apatite the mimetization process was used [11].

The encapsulation of PTX into the bio-NCP was performed by the double emulsion method, which allows for the inclusion of hydrophobic molecules into hydrophilic systems, i.e. PTX into a chitosan capsule [4]. Hereafter, the sample containing the PTX encapsulated into the bio-NCP will be referred as bio-NCP + PTX.

\subsection{Details on the experimental techniques}

PTX and bio-NCP + PTX structure was investigated by X-ray powder diffraction (XPD) using the D10B-XPD beamline at the Brazilian Synchrotron Light Laboratory (LNLS, Brazil) using $\lambda=1.24 \AA$.

This is an Open Access article distributed under the terms of the Creative Commons Attribution License 4.0, which permits unrestricted use, distribution, and reproduction in any medium, provided the original work is properly cited. 


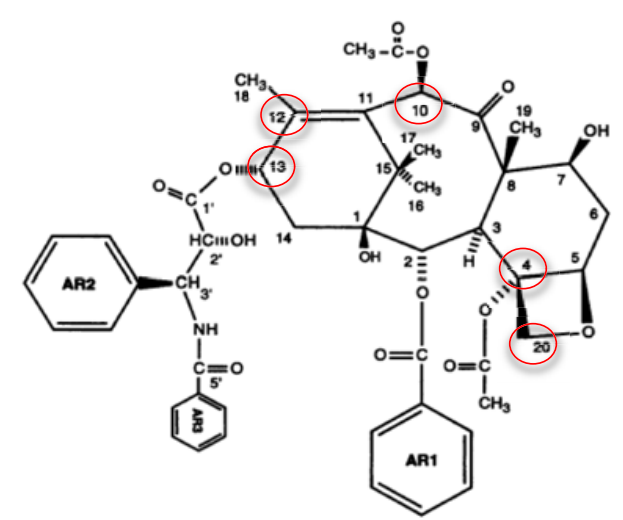

Figure 1. Schematic of the PTX molecule adapted from [10]. Circles highlight $\mathrm{C}$-atoms cited along the text.

To evaluate the solid-state properties of PTX, bioNCP, bio-NCP + PTX and of pure chitosan Thermal Gravimetric Analysis (TGA) and Differential Scanning Calorimetry (DSC) measurements were performed using a TG209 F1 Iris ASC, NETZSCH and a DSC (Q100)-TA instrument, respectively. The measurements were carried out between $120 \mathrm{~K}$ and $473 \mathrm{~K}$.

The dynamics of PTX, bio-NCP + PTX and the bio-NCP were further studied using FTIR, INS and QENS. FTIR measurements were carried out at room temperature between 400 and $4000 \mathrm{~cm}^{-1}$ using an ATR Crystal (Bruker). INS data were collected at IN4 (ILLFrance) at $10 \mathrm{~K}$ with incident neutrons of about $17 \mathrm{meV}$ $(\lambda=2.2 \AA)$ and $68 \mathrm{meV}(1.1 \AA)$. QENS and INS data were obtained with an incident wavelength $\lambda=5 \AA(3 \mathrm{meV}$, $\Delta \mathrm{E}=100 \mu \mathrm{eV}$ ) using the FOCUS spectrometer (PSISwitzerland). At FOCUS, PTX was measured at $100 \mathrm{~K}$, $150 \mathrm{~K}, 200 \mathrm{~K}, 250 \mathrm{~K}$ and $300 \mathrm{~K}$, while the bio-NCP and bio-NCP + PTX at $100 \mathrm{~K}, 200 \mathrm{~K}$ and $300 \mathrm{~K}$. The analysis of the temperature dependence of the elastic intensity of the PTX data allows for identifying relevant dynamical transitions. For the neutron experiments, the samples were mounted using the slab geometry inside of aluminum containers with a calculated normal beam transmission 0.75 . Vanadium runs were used to determine the resolution function and to calibrate the detectors efficiency. To analyze the data, the Bragg reflections were removed, the QENS spectra were fitted using the 1D PAN: Peak Analysis software [16] and the INS data were transformed into the generalized density of vibrational states using LAMP [17].

\section{Results and discussion}

\subsection{Structural characterization}

XPD pattern of PTX, Fig. 2a, shows two distinct crystalline phases attributed to a dehydrated and a hydrated form of the drug [19] with space groups P21212 and P1211, respectively. After the encapsulation, due to the loss of translation symmetry, only the ferrite and chitosan reflections can be observed in the bio-NCP + PTX pattern, Fig. 2b.

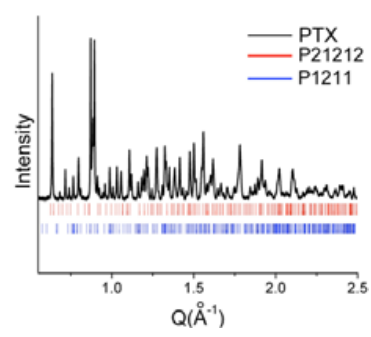

(a)

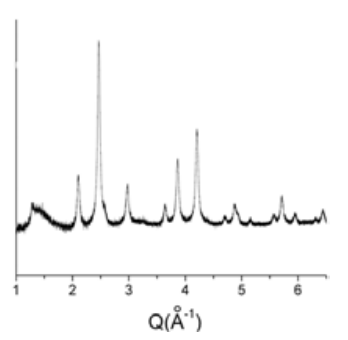

(b)
Figure 2. XPD of PTX (a) and bio-NCP + PTX (b) obtained at the LNLS using $\lambda=1.24 \AA$. The PTX pattern shows the presence of both hydrated and dehydrated semicrystalline forms, while in the the bio-NCP patterns only the reflections associated to the ferrite and chitosan are seen.

\subsection{Thermal analysis}

The DSC and TGA results between 300 and $473 \mathrm{~K}$ are shown in Figs. 3a and b. An extended phase transition is noted for PTX between 287 and $367 \mathrm{~K}$ accompanied by a tiny mass loss, while a second endothermic phenomenon is seen at $433 \mathrm{~K}$. The first phase transition refers to a partial dehydration of the compound inducing a structural rearrangement, while the second refers to a solid-solid transition [18]. These observations confirm that the analyzed PTX sample is a mixture of hydrated and dehydrated forms. For pure chitosan, Fig. 3a, an extended phase transition observed between 370 and $470 \mathrm{~K}$ is related to a water loss of about 3\% [19], while in the bio-NCP and bio-NCP + PTX this same transition is shifted to lower temperatures, i.e. between 340 and $435 \mathrm{~K}$ and $310 \mathrm{~K}$ and $430 \mathrm{~K}$, respectively. Due to confinement, the water loss in these samples occurs in two steps. As shown in the inset of Fig. 3a, in the bio-NCP the higher temperature step involves more energy than observed in the bio$\mathrm{NCP}+\mathrm{PTX}$. We can hypothesize that this is caused by a competition between the drug and the water molecules for interacting with the chitosan's $\mathrm{NH}$ and $\mathrm{OH}$ groups, thus reducing the material's hydration. To confirm this idea the samples were investigated by FTIR, as described below.

In the DSC data collected at lower temperatures, Fig. 3c, three subtle phase transitions centered at about 155,205 and $235 \mathrm{~K}$ are noted for the PTX, while no significant changes are observed for the other samples (data are not presented). Although such changes are very small in the DSC experiment, they might manifest themselves in the neutron scattering experiments [20].

\subsection{FTIR data analysis}

Regarding the FTIR data, Fig. 4, all the PTX vibrational peaks (black line) can be seen in the physical mixture (green line), while in the bio-NCP + PTX (red line) a considerable change in the spectra is noted. Furthermore, as shown in the inset of Fig. 4, the PTX bands at $1703 \mathrm{~cm}^{-1}$ from the $\mathrm{C}=\mathrm{O}$ ester stretching and at $1317 \mathrm{~cm}^{-1}$, assigned to the COO stretching [21] present in bio-NCP + PTX confirm the presence of PTX in the composite. Moreover, bending vibrations of NH groups of chitosan, around $1563 \mathrm{~cm}^{-1}$, as well as the vibrations of $\mathrm{OH}$ bonds in the primary groups of the polymer 


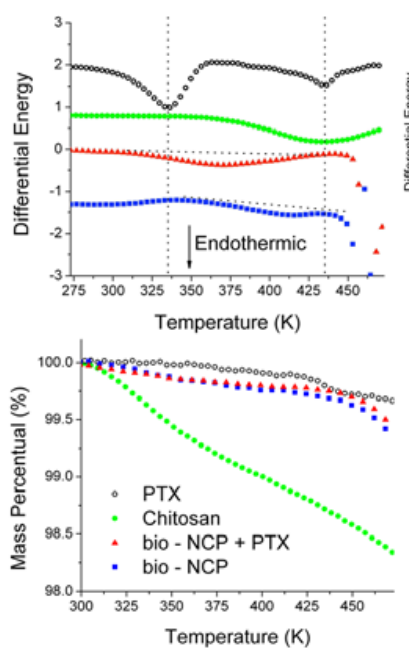

(b)

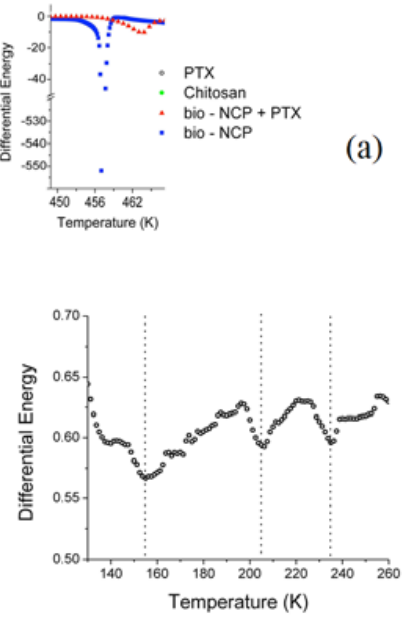

(c)
Figure 3. DSC analysis of PTX, bio-NCP, bio-NCP + PTX and pure chitosan between room temperature and $470 \mathrm{~K}$ (a). The inset shows a comparison between the events in bio-NCP and bio$\mathrm{NCP}+\mathrm{PTX}$ above $450 \mathrm{~K}$. In (b), TGA shows the water loss in the samples and (c) depicts the DSC analysis for PTX between $120 \mathrm{~K}$ and $300 \mathrm{~K}$.

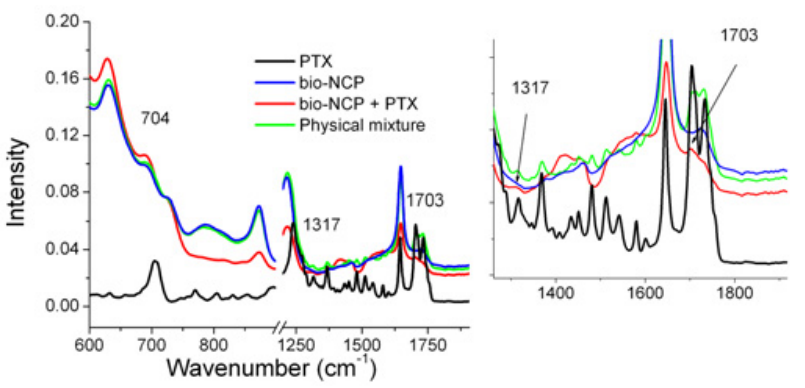

Figure 4. FTIR spectra for PTX (black), bio-NCP (blue), bioNCP + PTX (red) and physical mixture of bio-NCP and PTX (green). The inset highlights the presence of PTX peaks in the bio-NCP + PTX at $1317 \mathrm{~cm}^{-1}$ and $1703 \mathrm{~cm}^{-1}$.

around $1414 \mathrm{~cm}^{-1}$, [22] give rise to clear bands in the bio-NCP + PTX. Both observations corroborate with the DSC data, confirming the competition for interactions between PTX and the chitosan's NH and OH groups. Additionally, the prominent peak at $1650 \mathrm{~cm}^{-1}$ related to the C-N bonds, [23] is reduced in the bio-NCP + PTX. Since such bonds are formed during the cross-linking reaction and the formation of Schiff bases, which involve the $\mathrm{NH}$ groups, the reduction of this peak further reinforces the interaction of the drug with the chitosan.

\subsection{Neutron scattering: QENS and vibrational spectroscopy results}

Now we turn to the analysis of the temperature dependence of the low frequency spectra of pure PTX obtained on FOCUS, Fig. 5a. In the INS data four main broad bands centered at $80,150,103$ and $250 \mathrm{~cm}^{-1}$ (the latter almost out of the instrument limit) can be observed below $200 \mathrm{~K}$. Moreover, a clear change in the vibrational spectra is seen in the same temperature region where the first phase transition was observed in the DSC analysis. This change

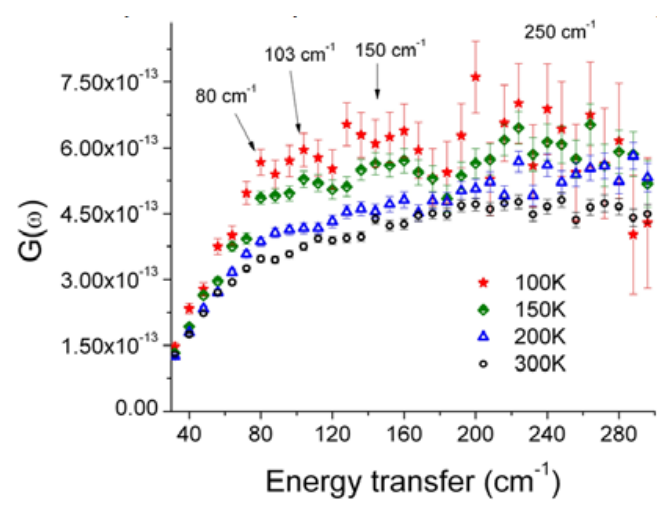

(a)

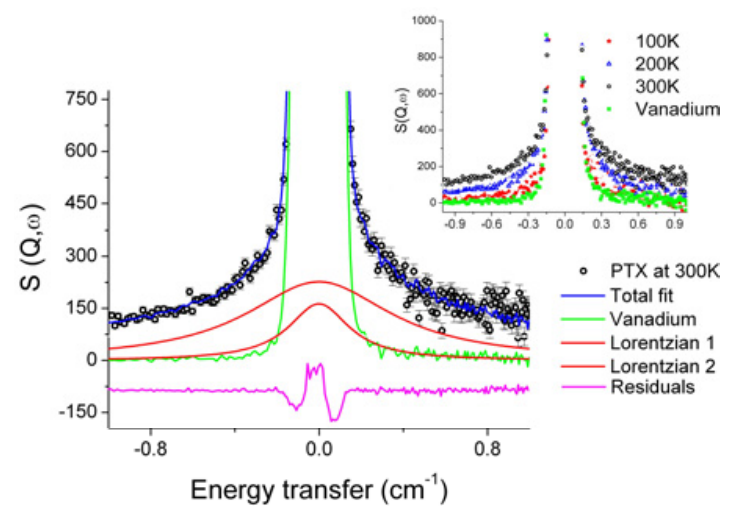

(b)

Figure 5. INS (a) and QENS data (b) obtained at $300 \mathrm{~K}$ for the pure PTX using FOCUS at the PSI. Above $100 \mathrm{~K}$ the data for PTX was fitted with two Lorentzian functions and one flat background. The inset depicts the QENS data obtained at selected temperatures, where a QE signal is visible.

is also accompanied by the observation of a quasi-elastic (QE) signal. To get insight on the activation energy of the processes related to the QE signal the data was fitted using a Dirac function and a sum of Lorentzians:

$$
S_{m}(Q, \omega)=\left[A_{0}(Q) \delta(\omega)+A_{i}(Q) \Sigma_{i} L_{i}\left(\Gamma_{i} \omega\right)\right] \otimes R(\omega)+B
$$

where $A_{i}$ gives the structure factors, $A_{0}$ is the elastic incoherent structure factor, $L_{\mathrm{i}}\left(\Gamma_{\mathrm{i}} \omega\right)$ are the Lorentzian functions (QE components), with a half-width at halfmaximum (HWHM) $\Gamma . \mathrm{R}(\omega)$ represents the experimental resolution function (vanadium run) and $\mathrm{B}$ the background.

In order to get a better statistics the signal was summed over the total Q-range. The data collected at $100 \mathrm{~K}$ could then be fitted using a single Lorentzian, while for all other temperatures an additional Lorentzian function was necessary to correctly describe the data, see Fig. 5.

Figure $6 \mathrm{a}$ shows that the temperature dependence of the integrated intensity under the elastic line for pure PTX reduces $\sim 19 \%$ (see the inset) between 100 and $300 \mathrm{~K}$, corresponding to the activation of $\sim 10 \mathrm{H}$ in the PTX molecule, or at least three $\mathrm{CH}_{3}$ groups. In addition, the changes in the slope of the curve at 150 and $200 \mathrm{~K}$ corroborate well with the phase transitions revealed by the DSC data and the changes in the inelastic part of the spectra. Therefore one can argue that the PTX molecule indeed undergoes some dynamical transitions in this temperature range. Figure $6 \mathrm{~b}$ depicts the Arrhenius 

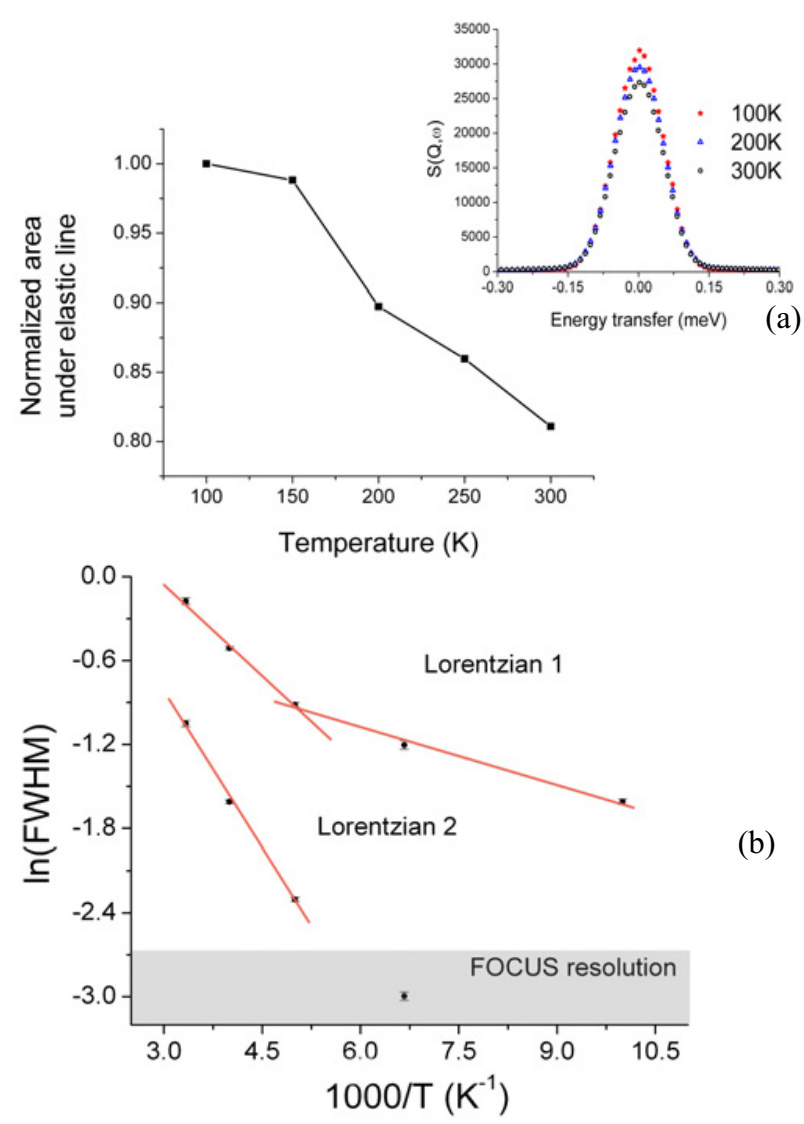

(b)

Figure 6. (a) Temperature dependence of the elastic line for pure PTX, shown in the inset for selected temperatures, integrated over the energy resolution interval. (b) Temperature dependence of the Full Width at Half Maximum (FWHM) of the QE signal observed for pure PTX obtained using the fitting procedure described in the text. The red lines indicate the Arrhenius model approximations. The error bars in (a) are smaller than the experimental points.

plot of the variation of the full width at half maximum (FWHM) for the drug molecule.

The relatively different values of the FWHM imply that in the PTX molecules re-orientational motions occur at a broad time scale. Using the Arrhenius law, activation energies of 1.16 and $3.6 \mathrm{~kJ} / \mathrm{mol}$ were obtained for the first Lorentzian, and $6.11 \mathrm{~kJ} / \mathrm{mol}$, for the second one. Assuming that the time scale of our QENS experiments probes mainly the dynamics of methyl groups contained in a harmonic potential with three-fold symmetry, it is possible to crudely estimate the $\mathrm{V}_{3}$ potential barrier as well as the first librational sub-state from the activation energy of these re-orientational motions using simplified numerical relations $\mathrm{E}_{0-1}(\mathrm{meV})=0.47\left[\mathrm{~V}_{3}(\mathrm{~K})\right]^{0.548}$ and $\mathrm{E}_{\text {act }}(\mathrm{K})=0.598\left[\mathrm{~V}_{3}(\mathrm{~K})\right]^{1.05}[20]$. If this assumption is suitable, one would expect librational peaks around 64, 96 and $144 \mathrm{~cm}^{-1}$. Based on our ongoing DFT calculations (private communication) [24], the two lower vibrations are mainly attributed to the acetyl groups bonded to $\mathrm{C} 4$ and $\mathrm{C} 10$ and the methyl group in C12, respectively (Fig. 1), calculated at 66 and $96 \mathrm{~cm}^{-1}$. Differences between the theoretical and experimental values as well as the broadening in the observed peaks in Fig. 5a denote that

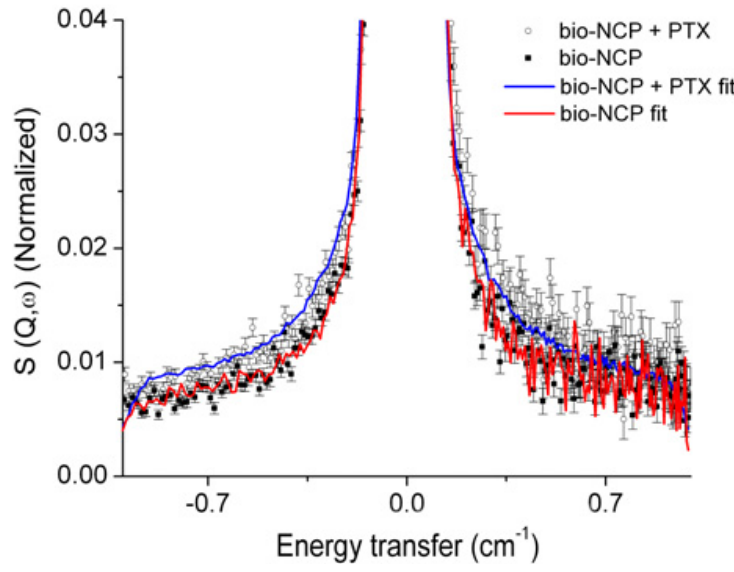

Figure 7. Normalized $\mathrm{S}(\mathrm{Q}, \omega)$ spectra of the bio-NCP and bio$\mathrm{NCP}+\mathrm{PTX}$ at $300 \mathrm{~K}$. The results for the fits of the QE signal are also shown.

the methyl groups are not in a pure $\mathrm{C}_{3}$ symmetry. For the lowest energy the side chain in C13 also plays an important role. Considering the higher energy vibration, calculated at $143 \mathrm{~cm}^{-1}$, a contribution from C20 in the oxetane ring explains the broadening in the observed data.

Regarding the QENS spectra of the bio-NCP and bio-NCP + PTX the data collected at $100 \mathrm{~K}$ and $200 \mathrm{~K}$ could be described using a single Lorentzian and a flat background function, while for fitting the bio-NCP + PTX data collected at $300 \mathrm{~K}$ an additional Lorentzian was necessary. No differences in the QE broadening could be noted at $100 \mathrm{~K}$ and $200 \mathrm{~K}$, suggesting the strong confinement of the PTX inside the bio-NCP. However, as in seen Fig. 7, a slight difference between the bio$\mathrm{NCP}$ and bio-NCP + PTX can be observed at $300 \mathrm{~K}$, thus allowing insight into the contribution from the drug itself. A different background offset was needed due to the increasing contribution of the Debye-Waller factor, and for the additional Lorentzian a FWHM value of $0.72 \mathrm{meV}$ was obtained. Considering that this value is close to the one found for the first Lorentzian used to fit the PTX data, $0.84 \mathrm{meV}$, we suggest that the extra QE signal observed in the bio-NCP + PTX spectrum at $300 \mathrm{~K}$ might be assigned to the drug. However, the validation of this idea needs further studies.

Insight in the dynamics between 20 and $480 \mathrm{~cm}^{-1}$ was by analyzing the IN4 data. As shown in Fig. 8, the band centered at $48 \mathrm{~cm}^{-1}$ in the PTX spectrum is damped in the bio-NCP + PTX. In this region, the DFT calculations show a great contribution from the PTX phenyl rings and acetyl groups, which therefore seem to be constrained by encapsulation. In addition, small contributions around $110 \mathrm{~cm}^{-1}$ are noted in the difference curve obtained by subtracting the bio-NCP + PTX signal from that collected for the bio-NCP (green curve). Small contributions in the difference curve are further noted in the data collected using incident neutrons with $67.6 \mathrm{meV}$ energy around $280 \mathrm{~cm}^{-1}, 330 \mathrm{~cm}^{-1}$ and above $400 \mathrm{~cm}^{-1}$. Such contributions indicate that the encapsulated drug preserves some of its vibrations. 

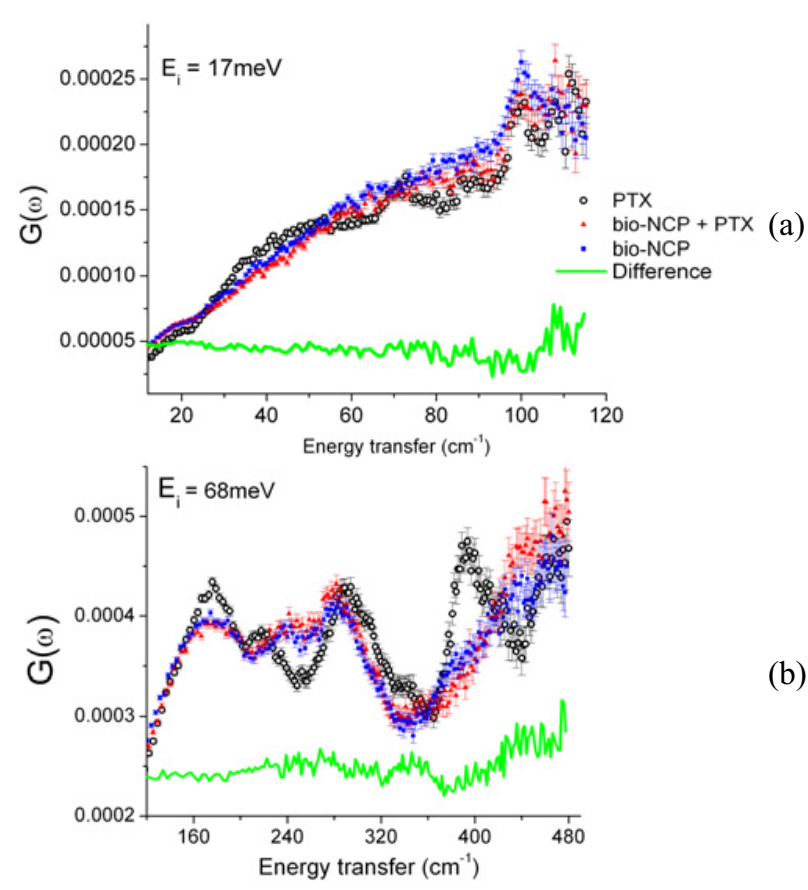

Figure 8. INS spectra collected using IN4 at $10 \mathrm{~K}$ for PTX (black circles), bio-NCP + PTX (red triangles) and bio-NCP (blue squares). The green curve shows a difference between bioNCP + PTX and bio-NCP using incident energies of 17 (a) and $68 \mathrm{meV}$ (b).

\section{Conclusions and discussion}

Typically thermal analysis methods are considered to be the most suitable approaches for determination of a successful encapsulation process. Normally, these techniques are supplemented by FTIR to show that the loss of the melting peak of the pure drug is due to its complexation rather than thermal degradation or a loss of crystallinity. Generally, the bands due to the complexed part of the drug are shifted or have their intensities altered. However as the mass of the encapsulated drug does not exceed $5-15 \%$ of the complex, these alterations are usually hidden, and only in some cases spectroscopic changes indicating complex formation can be observed. In addition, in the case of crystalline drugs such as PTX studied in this paper, encapsulation leads to a disruption of the intermolecular hydrogen bonding of the pure drug. Consequently the Bragg reflections characteristic of the isolated molecules are absent in the XPD patterns of the encapsulated molecule, and only the Bragg reflections characteristics of the encapsulation itself can be observed. Owing to the same reason, intermolecular vibrations cannot be observed in the complexed system, and no useful information is acquired in the far IR region.
Here we put forward a new idea that by using INS further insight on the local dynamics of the pure drug molecule and how its dynamics is affected by the encapsulation can be obtained. Since neutron scattering is not a surface technique and the sample does not need to be manipulated in order to obtain the data, this probe is extremely suitable for confinement studies. Additionally, we presented results following the classical approach to confirm that PTX was encapsulated into the bio-NCP.

Our results, even if in a very early stage, are encouraging. Using neutron scattering we show that a number of vibrational modes are damped indicating that the PTX molecule is constrained by the encapsulation. Moreover from the analysis of the QENS data we were able to obtain valuable information on the flexiblility of the PTX molecular groups, and how this flexibility might be modified by encapsulation in the drug carrier bio-NCP + PTX. Further analyses are under way and will be discussed in a future publication.

MLM work was financed by the Brazilian Science without borders program. Measurements at the Swiss spallation neutron source SINQ has received funding under the NMI3-II Grant number 283883 (MLM), while the Danscatt program financed the experiments carried out at the ILL (MLM and HNB).

\section{References}

[1] C. Wang, et al., Int. J Pharm, 400, 201(2010)

[2] X. Yu, M.V. Pishko. Biomacromol, 12, 3205 (2011)

[3] A. Camirand, et al. Am J Cancer Res., 3, 500 (2013)

[4] P. Lv, et al., Biomacromol, 12, 4230 (2011)

[5] R. Meena, et al., J. Nano. Res. 14, 712 (2012)

[6] C. Alexiou et al., Eur Biophys J, 35, 446 (2006)

[7] A.I. Hilger et al., J Magn. Magn. Mater, 293, 314 (2005)

[8] T. Moraes et al., Phyisiol. Meas., 24, 337 (2003)

[9] P.M. Ferguson. Plos one, 8, 2 (2013)

[10] D. Mastropaolo et al. P.N.A.Sci. 92, p. 6920 (1995)

[11] M.L. Martins et al. J Alloys Compd, 584, 514 (2014)

[12] Y. Li et al., Biochemistry, 39, 281 (2000)

[13] H.J. Williams, et al., Tetrahedron, 49, 30, 6545 (1993)

[14] J.K. Harper et al., Cryst Growth Des, 5, 1737 (2005)

[15] M.L. Martins et al., Appl. Clay Sci. 96, 22 (2014)

[16] R.T. Azuah, et al., J. Res. NIST. 114, 341 (2009)

[17] D. Richard, et al., J. Neutron Research 4, 33 (1996)

[18] R.T. Liggins et al. J Pharm Sci, 86, 1458 (1997)

[19] C.G.T. Neto et al., Carb. Polymers, 62, 97 (2005)

[20] H.N. Bordallo et al., Biophys Chem, 148, 34 (2010)

[21] S. Alipour et al., Colloids Surf B, 81, 521 (2010)

[22] A. Sionkowska et al., Biomaterials, 25, 795 (2004)

[23] J. Klinkaewnarong et al. App. Phys. 10, 521 (2010)

[24] M.L. Martins and J. Eckert, private communication 